\title{
Pilot capability evaluation of a feedback electronic imaging system prototype for in-process monitoring in electron beam additive manufacturing
}

\author{
Hay Wong ${ }^{1}$ (i) $\cdot$ Derek Neary $^{1} \cdot$ Eric Jones $^{2} \cdot$ Peter Fox $^{1} \cdot$ Chris Sutcliffe $^{1}$
}

Received: 29 April 2018 / Accepted: 11 September 2018 / Published online: 27 September 2018

(C) The Author(s) 2018

\begin{abstract}
Electron beam additive manufacturing (EBAM) is an additive manufacturing (AM) technique increasingly used by many industrial sectors, including medical and aerospace industries. The application of this technology is still, however, challenged by many technical barriers. One of the major issues is the lack of process monitoring and control system to monitor process repeatability and component quality reproducibility. Various techniques, mainly involving infrared (IR) and optical cameras, have been employed in previous attempts to study the quality of the EBAM process. However, all attempts lack the flexibility to zoom-in and focus on multiple regions of the processing area. In this paper, a digital electronic imaging system prototype and a piece of macroscopic process quality analysis software are presented. The prototype aims to provide flexibility in magnifications and the selection of fields of view (FOV). The software aims to monitor the EBAM process on a layer-by-layer basis. Digital electronic images were generated by detecting both secondary electrons (SE) and backscattered electrons (BSE) originating from interactions between the machine electron beam and the processing area using specially designed hardware. Prototype capability experiments, software verification and demonstration were conducted at room temperature on the top layer of an EBAM test build. Digital images of different magnifications and FOVs were generated. The upper range of the magnification achieved in the experiments was 95 and the demonstration verified the potential ability of the software to be applied in process monitoring. It is believed that the prototype and software have significant potential to be used for in-process EBAM monitoring in many manufacturing sectors. This study is thought to be the necessary precursor for future work which will establish whether the concept is suited to working under in-process EBAM operating conditions.
\end{abstract}

Keywords Additive manufacturing · Electron beam melting · Metallic materials · In-process monitoring · Quality control · Electronic imaging $\cdot$ Secondary electrons $\cdot$ Backscattered electrons

\section{Introduction}

Electron beam additive manufacturing (EBAM) is an additive manufacturing (AM) technique that makes use of an accelerated electron beam to melt metallic powder on a layer-by-layer basis, manufacturing components based on computer-aided design (CAD) models [1]. The ability of the EBAM process to form components from metallic powder arises from

Hay Wong

hay.wong@liv.ac.uk

1 School of Engineering, University of Liverpool, The Quadrangle, Brownlow Hill L69 3GH, UK

2 Jones Consultancy, Ardlahan, Kildimo, Co. Limerick, Ireland electron interactions with metallic materials. In the EBAM process, an electron beam is accelerated by an anode, focused onto a powder bed by an electromagnetic focusing coil and subsequently deflected to specific locations by an electromagnetic deflection coil, the electrons penetrate the powder grains, whereupon they decelerate, converting their kinetic energy into thermal energy. If the energy input is sufficient, the temperature of the powder particles rises above their melting point and the particles melt. When the beam is raster-scanned across the preheated powder bed in a tightly controlled, predefined pattern, melt tracks are solidified to form fully dense cross sections of the desired single or multi-components constituting the build. This process is repeated with the additional requirement that the underlying solid is also partially melted to ensure adequate bonding between the underlying and newly formed layers, ensuring that full density is achieved [1]. 
Table 1 Thermal and optical monitoring systems developed for the EBAM process

\begin{tabular}{|c|c|c|c|c|}
\hline $\begin{array}{l}\text { Process attribute } \\
\text { monitored }\end{array}$ & $\begin{array}{l}\text { Monitoring } \\
\text { method }\end{array}$ & $\begin{array}{l}\text { Process } \\
\text { artefacts }\end{array}$ & Sensor & Reference \\
\hline $\begin{array}{l}\text { Processing area } \\
\text { surface temperature }\end{array}$ & Thermal imaging & IR & CCD sensor & [8-13] \\
\hline Melt pool geometry & $\begin{array}{l}\text { Thermal / optical } \\
\text { imaging }\end{array}$ & $\begin{array}{l}\text { IR / visible } \\
\text { light }\end{array}$ & $\begin{array}{l}\text { Microbolometer / } \\
\text { CMOS sensor }\end{array}$ & {$[11,14]$} \\
\hline Component geometry & Thermal imaging & IR & Microbolometer & {$[15]$} \\
\hline Porosity in component & Thermal imaging & IR & Microbolometer & {$[16-20]$} \\
\hline $\begin{array}{l}\text { Electron beam profile and } \\
\text { position }\end{array}$ & $\mathrm{X}$-ray detection & X-ray & $\begin{array}{l}\text { Semiconductor } \\
\text { detector }\end{array}$ & {$[21]$} \\
\hline
\end{tabular}

EBAM shows great promise in the manufacture of orthopaedic implants and aerospace components. In an evaluation study on powder-based EBAM technology, it has been concluded that the EBAM process would enable the manufacture of a wide range of complex and difficult-to-fabricate aerospace and biomedical components [2]. The increased design freedom of the EBAM process enables the economic manufacture of porous bone ingrowth surfaces for orthopaedic implants [3] whilst the reduced thermal residual stress and the high-vacuum process environment is beneficial for the production of aircraft components [4]. Nevertheless, both of these industries are highly regulated and their current standard manufacturing processes are well established [5, 6]. Despite the perceived benefits of the EBAM process, the transition from current standard manufacturing techniques to a layered manufacturing approach will not be possible unless many current technology gaps, including having a rigorous in-process monitoring and validation system for realtime control of the EBAM process, are bridged [7].

Academic research groups have built various monitoring systems to assess the quality of the EBAM process. These systems are thermal/optical imaging-based, involving the use of either IR (wavelength between $700 \mathrm{~nm}$ and $1 \mathrm{~mm}$ ), visible light (wavelength between $400 \mathrm{~nm}$ and $700 \mathrm{~nm}$ ) or X-ray (wavelength between $0.01 \mathrm{~nm}$ and $10 \mathrm{~nm}$ ). Table 1 summarises the monitoring systems developed.

Each of the systems reported in Table 1 has many useful features; however, significant limitations exist in all current systems. Table 2 summarises these limitations with particular emphasis on the requirements of EBAM.
We postulate that the use of a digital electronic imaging system, following the methodology used in electron beam welding (EBW) and digital scanning electron microscopes (SEMs), would tackle the major functional inadequacies in existing thermal/optical data collection systems described previously.

EBW is a technique which processes materials with a focused, accelerated electron beam. EBW is commonly used for joining metals (including refractory metals) due to the available concentrated thermal energy, up to $10^{8} \mathrm{~W} / \mathrm{cm}^{2}$ [27], delivered by the electron beam. During EBW, process artefacts including secondary electrons (SE), backscattered electrons (BSE) and/or electrons in the plasma plume are generated above the welding zone [28]. By capturing some or all of these artefacts, electronic images [27, 29] and electron signal-time series plots [28-31] can be generated post and during EBW. Electron beam focus quality [28] and weld-quality attributes including weld-seam quality [27] and keyhole depth [32] are commonly monitored in industry.

In thermal and optical imaging, the adjustments of FOV and magnification are either not possible or involve movements of physical lenses. On the contrary, when conducting electronic imaging (by the collection of SE and/or BSE) with a SEM, the change in FOV and magnification only requires scanning the primary electron beam over a different region of interest (ROI) [33]. Given this flexibility and the ability for electronic imaging to reveal the topography of a conductive area [34], the incorporation of an electronic imaging system in the EBAM process will bridge the technology gap and contribute to the effective live EBAM process monitoring.
Table 2 Major function inadequacies in monitoring porosity and component geometry

\begin{tabular}{|c|c|c|}
\hline Inadequacy & Description & Reference \\
\hline Long imaging time & $\begin{array}{l}\text { Shields are required to protect system sensors from heat and } \\
\text { metallisation from the EBAM process. Movements of shields } \\
\text { increase imaging time }\end{array}$ & {$[22]$} \\
\hline $\begin{array}{l}\text { Unable to exclude } \\
\text { irrelevant area }\end{array}$ & $\begin{array}{l}\text { Monitoring-irrelevant areas lead to an unnecessary increase in dataset } \\
\text { size and data processing time }\end{array}$ & {$[8,10,23]$} \\
\hline Inflexible FOV & $\begin{array}{l}\text { Only capable of either monitoring the whole processing area or one } \\
\text { fixed region }\end{array}$ & [24-26] \\
\hline $\begin{array}{l}\text { Inflexible } \\
\text { magnification }\end{array}$ & $\begin{array}{l}\text { Unable to switch from monitoring the full processing area to zoom-in to } \\
\text { specific regions }\end{array}$ & {$[24]$} \\
\hline
\end{tabular}


Fig. 1 Schematic of the a electronic imaging system prototype [35], b electron sensor, c signal differential amplifier and d data logger

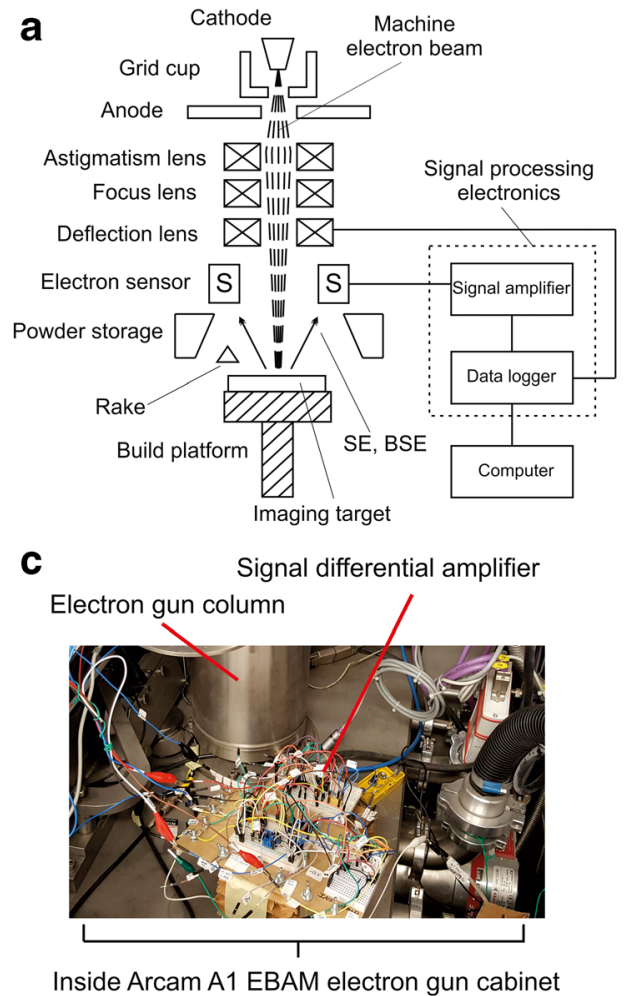

\section{Electronic imaging system design and experiments}

\subsection{Electronic imaging system prototype}

A bespoke digital electronic imaging system prototype [35], shown in Fig. 1a, was used for experiments investigating image magnification and FOV. The in-house developed prototype consists of an electron sensor (Fig. 1b), a signal differential amplifier (Fig. 1c), data logger (Fig. 1d), an image generation software and a standard computer. It was designed to generate digital images from the secondary electrons (SE) and backscattered electron (BSE), hereafter collectively referred to as feedback electrons, originating from the interactions between a primary electron beam and an imaging target. The electron sensor was made of electronically conductive materials; amplifier and data logger were put together with off-the-shelf, standard electronic components and an
Fig. 2 a The completed test build manufactured by the EBAM machine with Ti-6Al-4V powder of $45 \mu \mathrm{m}-106 \mu \mathrm{m}$ in size, with nine imaging locations [35]. b The EBAM machine processing platform, where the test build was placed on top during experiments. Note that there are no fixation points to position the build precisely a

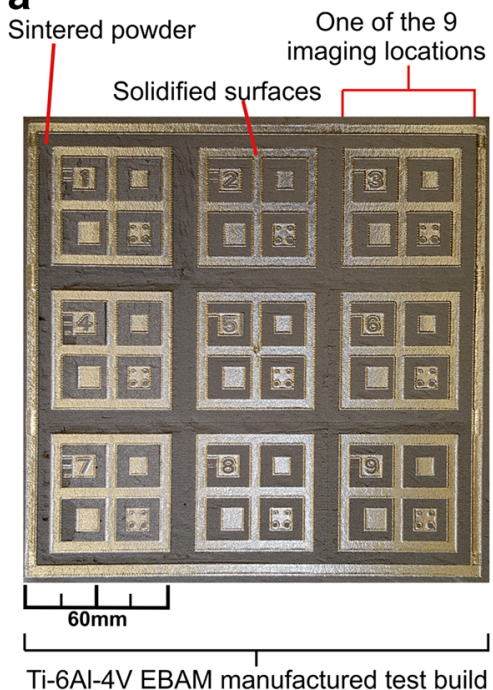

b

Processing area height adjusting wheels

Type K thermocouple

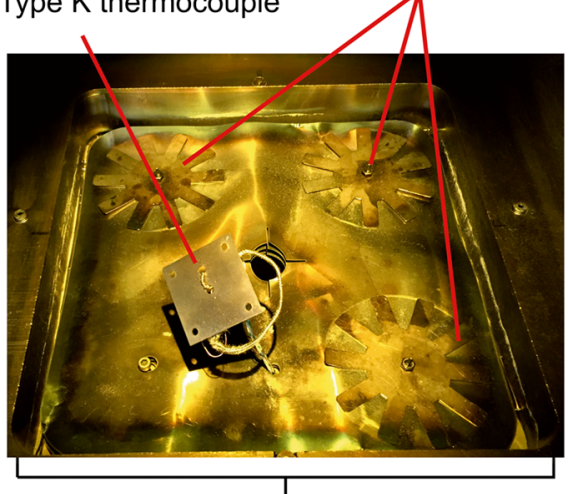

Arcam A1 EBAM machine processing area

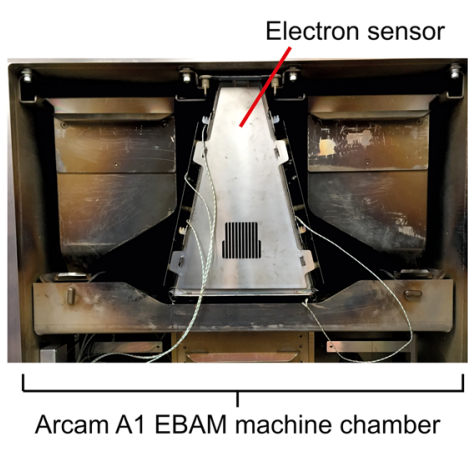
signal processing circuit

Data logger 
Fig. 3 Design, EBAM build setup and the manufactured hip prostheses [36]
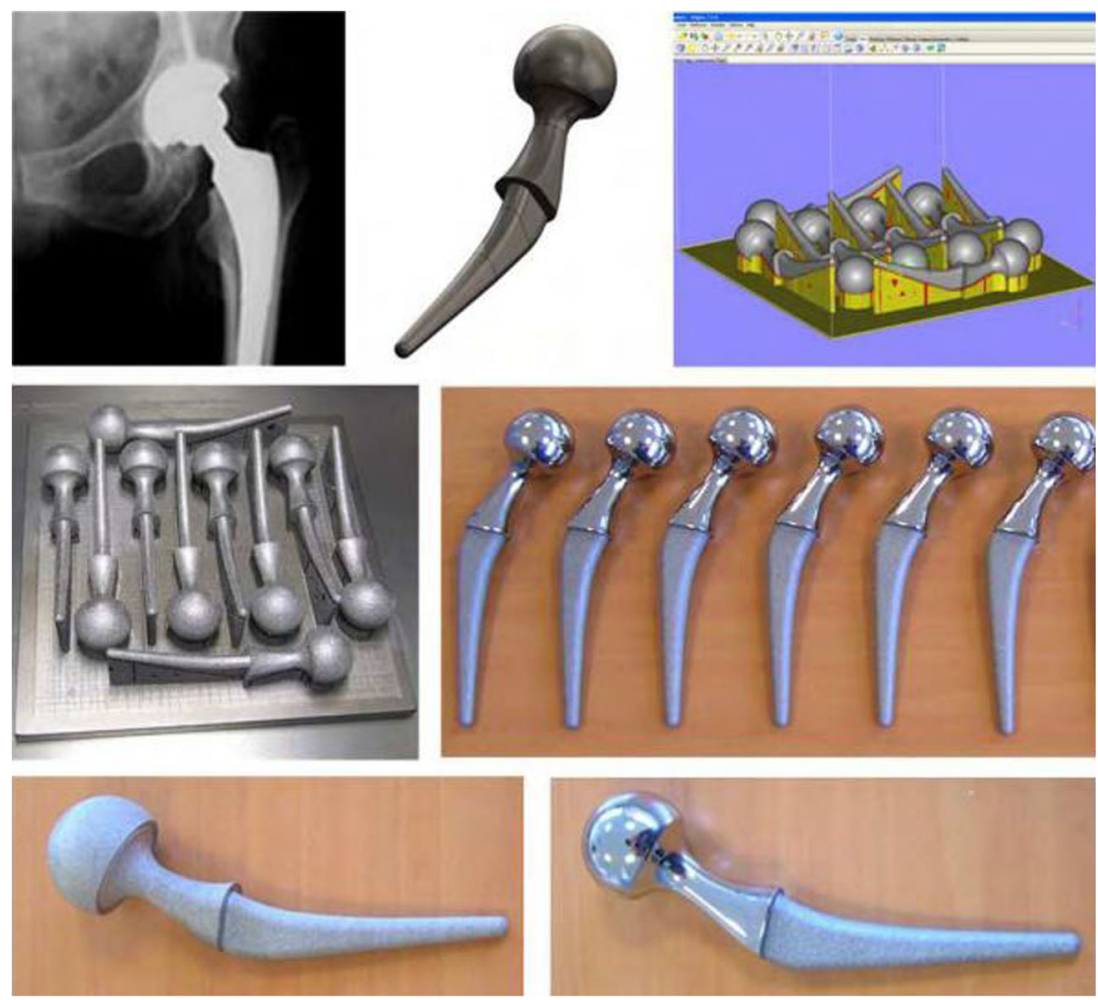

Arduino DUE microcontroller break-out board. Electron sensor signals were taken out from the EBAM machine chamber via a vacuum feedthrough and communication between the electronics and computer was carried out with Universal Serial Bus (USB) 2.0 industrial standard protocol.

\subsection{Experimental arrangements}

The prototype was interfaced with a commercial EBAM machine (Arcam A1-GE Additive, USA), and two sets of singlelayer electronic imaging experiments were conducted at room temperature. The first set aimed to generate images with a range of magnifications whilst the second concerned image FOV. The two sets of experiments were carried out on a standard electron imaging test build [35] shown in Fig. 2. The test build was designed to investigate various electronic imaging abilities including image contrast, magnification and spatial resolution. The tests build contained gaps to trap sintered

Table 3 Electronic imaging system prototype settings

\begin{tabular}{ll}
\hline Prototype parameter & Value \\
\hline Signal differential amplifier gain & 10 \\
Data logger sampling frequency & $118.8 \mathrm{kHz}$ \\
Data logger input/output range & $0 \mathrm{~V}-+3.3 \mathrm{~V}$ \\
Image size & 1800 pixel $\times 1800$ pixel \\
Image bit depth & $8-$ bit, 256 \\
\hline
\end{tabular}

powder and contained geometric features including squares and holes for image features extraction purposes. The test build was designed to have nine imaging locations, each with a dimension of $60 \mathrm{~mm} \times 60 \mathrm{~mm}$, representing nine virtual components packed into an EBAM processing area of $210 \mathrm{~mm} \times 210 \mathrm{~mm}$. In a typical industrial EBAM build, multiple components are packed into the processing area to maximise productivity; Fig. 3 shows an example of an EBAM hip prosthesis design and its EBAM build setup extracted from the literature [36].

This paper focuses on (1) image magnification and (2) image FOV. The geometric features of the test build will not be discussed in this study. Table 3 details the settings of the prototype whilst Table 4 gives the EBAM machine settings and the range of monitoring areas involved in the two sets of experiments. The electron beam current and scan speed presented in Table 4 were dictated by the signal amplifier gain, data logger sampling rate and input range

Table 4 EBAM machine primary electron beam settings and imaging areas

\begin{tabular}{llcl}
\hline Imaging area $\left(\mathrm{mm}^{2}\right)$ & Current $(\mathrm{mA})$ & Speed $\left(\mathrm{mms}^{-1}\right)$ & Focus offset $(\mathrm{mA})$ \\
\hline $180 \times 180$ & 1 & 11,880 & 0 \\
$60 \times 60$ & 1 & 3960 & 0 \\
$10 \times 10$ & 0.5 & 660 & 0 \\
$5 \times 5$ & 0.5 & 330 & 0 \\
\hline
\end{tabular}


Table 5 Magnification and FOV tests setup

\begin{tabular}{lll}
\hline Investigation & Imaging location & Imaging area $\left(\mathrm{mm}^{2}\right)$ \\
\hline Magnification & 5 & $180 \times 180,60 \times 60,10 \times 10,5 \times 5$ \\
FOV & $1-9$ & $60 \times 60$
\end{tabular}

described in Table 3. The imaging areas were chosen based on the design of the test build, i.e. dimension and design of the imaging locations.
For the evaluation of image magnification, the machine primary electron beam raster-scanned across a range of imaging areas across imaging location 5 of the test build. When investigating the FOV, the beam raster-scanned across different ROIs with the same imaging area, covering the full machine processing area. Table 5 gives the magnification and FOV tests setup. With regard to trials on image FOV, only the $60 \mathrm{~mm} \times 60 \mathrm{~mm}$ image area was chosen to be involved in this pilot FOV experiment as a demonstration of the prototype capability. Based on the test build depicted in Fig. 2a, a
Fig. 4 STL image generation and macroscopic process quality analysis software process flow for an arbitrary layer

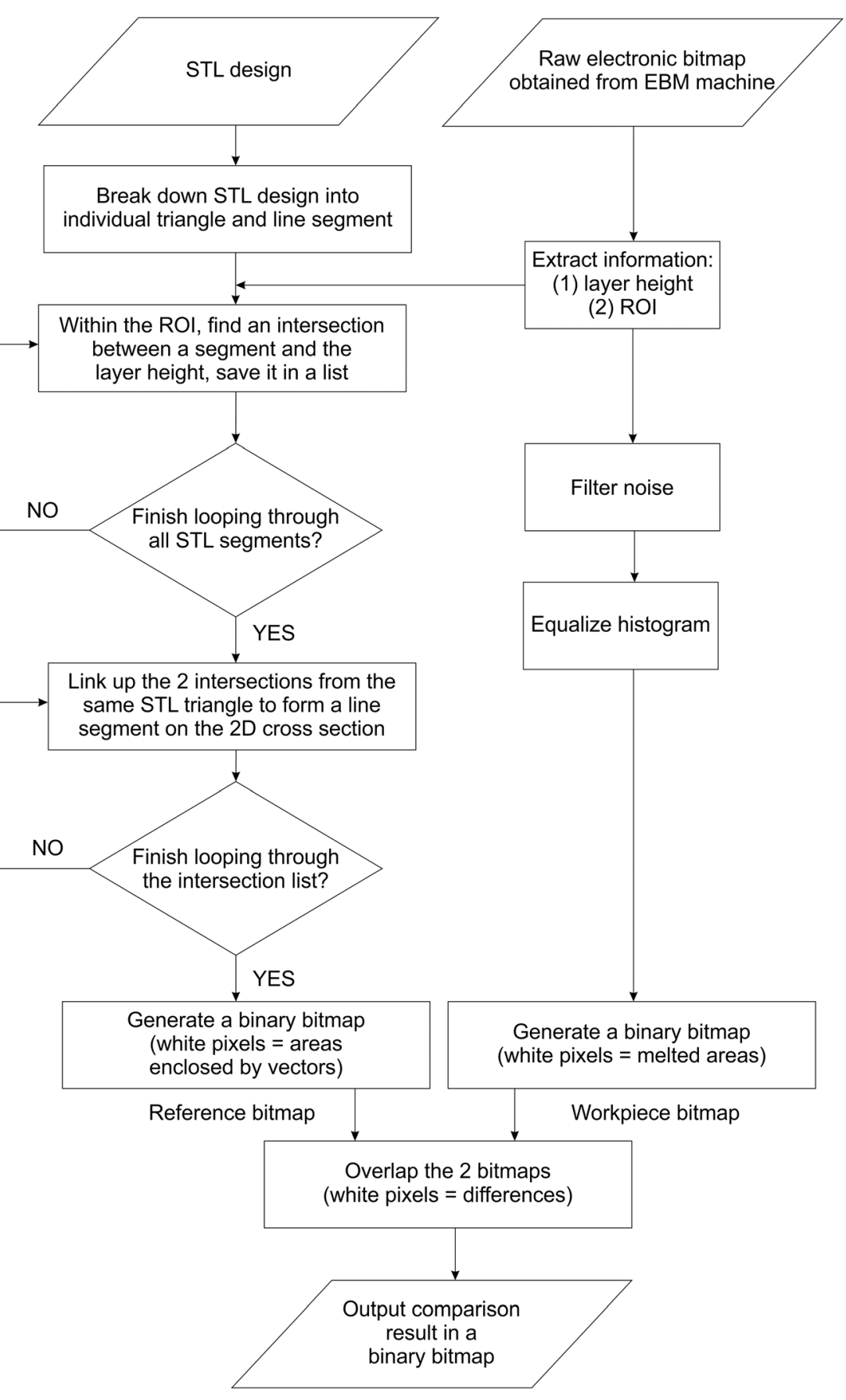


Sub-surface "bridge" structures will be covered by sintered Ti-6Al-4V powder

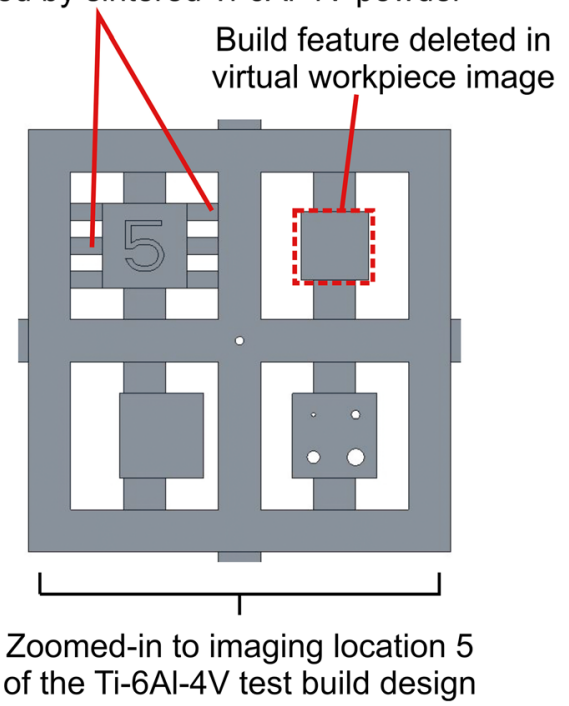

Fig. 5 Virtual workpiece image design for the macroscopic process quality analysis software verification

$60 \mathrm{~mm} \times 60 \mathrm{~mm}$ area would show the imaging location labels, indicating clearly the selection of different image FOVs by the system prototype.

\subsection{Macroscopic process quality analysis}

This section presents the development, verification and demonstration setup of an image quality analysis software.

\subsubsection{Software development}

In a typical EBAM build, multiple components are packed into the processing area to maximise productivity. Carrying out EBAM process monitoring with electronic imaging opens up the opportunity to monitor individual areas of components by zooming in to the corresponding ROI to achieve the

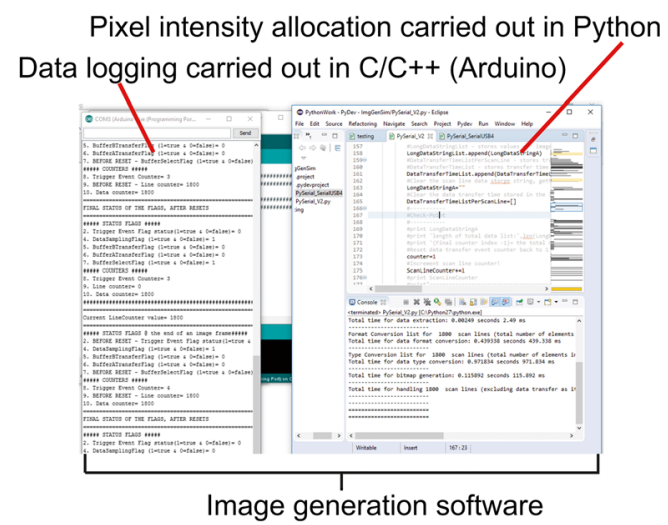

Fig. 6 Computer screenshot showing image generation software in operation
Table 6 Typical image generation time reported by image generation software, data rounded to 3 s.f

\begin{tabular}{ll}
\hline Image generation procedure & Time involved (ms) \\
\hline Scan line transfer & 74.3 \\
Data extraction & 1.93 \\
Data format conversion & 387 \\
Data type conversion & 909 \\
Total time for image generation & $1370(1.37 \mathrm{~s})$ \\
\hline
\end{tabular}

required FOV. A piece of software was developed in an open-source programming language, Python, aiming to assess individual component quality on a layer-by-layer basis on a macroscopic scale. Only the bulk quality was designed to be assessed, i.e. detailed component geometry with edge detection was not involved. This macroscopic quality analysis targeted process anomalies and defects from over-melting, peeled-off metallisation [37] and lack of powder deposition in the local processing area.

At the beginning of the analysis, the software generated a stack of two-dimensional (2D) reference images from a threedimensional (3D) stereolithography (STL) design according to layer height and user-defined ROI. It then compared the reference images with the workpiece images from the same layer height and ROI generated by the electronic imaging system prototype. In order to evaluate the macroscopic process quality, both the reference and workpiece images were first binarised to form black and white images. Comparison between these two images was made and the differences between the two were expressed as white pixels, in the resultant image. Bulk process quality was quantitatively assessed by evaluating the ratio of the number of white pixels to that of black pixels in the resultant binary image. Figure 4 describes the software process flow for an arbitrary layer.

\subsubsection{Software verification}

Once the software was developed, a benchtop verification experiment was carried out to prove the correct design and operation of the software. In the experiment, a verification "reference-virtual workpiece" image set was prepared. The imaging location 5 area from the Ti-6Al-4V test build design was chosen to be the ROI for verification. A reference image was generated from this location and a virtual workpiece image was generated from the same ROI with the $8 \mathrm{~mm} \times 8 \mathrm{~mm}$ square on the top right-hand corner removed artificially. Figure 5 depicts how this virtual workpiece image was generated from the imaging location 5 test build design. Macroscopic process quality analysis was conducted on the "reference-virtual workpiece" image set. The virtual workpiece image was overlapped onto the reference image and the difference between the images was evaluated and shown as white pixels in a resultant image. If the 
Table 7 Magnifications calculated for images covering different imaging areas, based on a 96 dots per inch (DPI) computer monitor

\begin{tabular}{llll}
\hline Monitoring area size $\left(\mathrm{mm}^{2}\right)$ & Image size $\left(\mathrm{pixel}^{2}\right)$ & Image size $\left(\mathrm{mm}^{2}\right)$ & Magnification $(2$ s.f. $)$ \\
\hline $180 \times 180$ & $1800 \times 1800$ & 476.25 & 2.6 \\
$60 \times 60$ & $1800 \times 1800$ & 476.25 & 8.0 \\
$10 \times 10$ & $1800 \times 1800$ & 476.25 & 48 \\
$5 \times 5$ & $1800 \times 1800$ & 476.25 & 95 \\
\hline
\end{tabular}

measured number of white pixel in the resultant image corresponded to the expected number of pixels occupied by the $8 \mathrm{~mm} \times 8 \mathrm{~mm}$ square, this benchtop experiment would verify the correction operation of the software.

\subsubsection{Software demonstration}

A demonstration trial was carried out with the test build depicted in Fig. 2a. The demonstration aimed to showcase the software ability to monitor a local ROI, i.e. the individual imaging locations 1-9 (representing individual component in a real EBAM build). Upon completion, the test build was removed from the EBAM chamber with subsequent removal of excess sintered powder. The test build was replaced in the chamber and further electronic imaging was carried out with the prototype as shown in Fig. 1a-d. During imaging, the electron beam raster-scanned across the $60 \mathrm{~mm} \times 60 \mathrm{~mm}$ ROI of location 5 and a workpiece image revealing the real topographical features of location 5. A reference image covering the same ROI was extracted from the top layer of the test build STL design by the software. The software then carried out macroscopic process quality analysis to evaluate the quality of the top layer by overlapping the workpiece image onto the reference image.

\section{Results}

This section presents the experimental results of electronic image generation and macroscopic process quality analysis.
Fig. 71800 pixel $\times 1800$ pixel electronic digital images (processed) covering imaging areas of different sizes across the standard electronic imaging test build. a $180 \mathrm{~mm} \times 180 \mathrm{~mm}$. b $60 \mathrm{~mm} \times 60 \mathrm{~mm}$. c $10 \mathrm{~mm} \times$ $10 \mathrm{~mm}$. d $5 \mathrm{~mm} \times 5 \mathrm{~mm}$ a

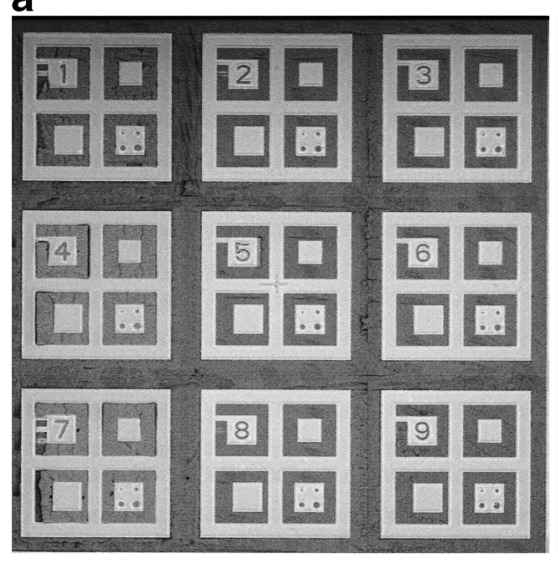

c

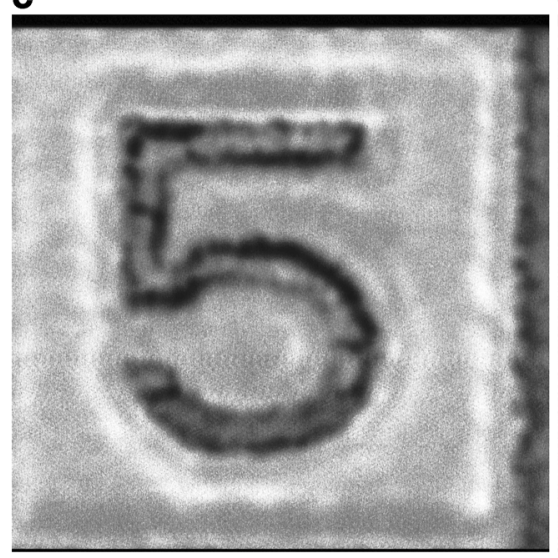

b

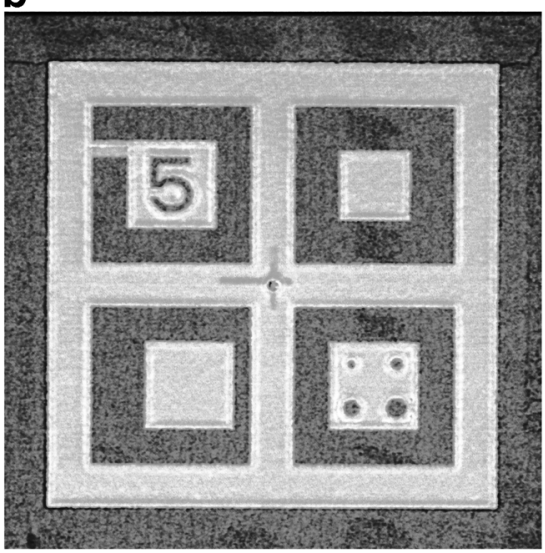

d

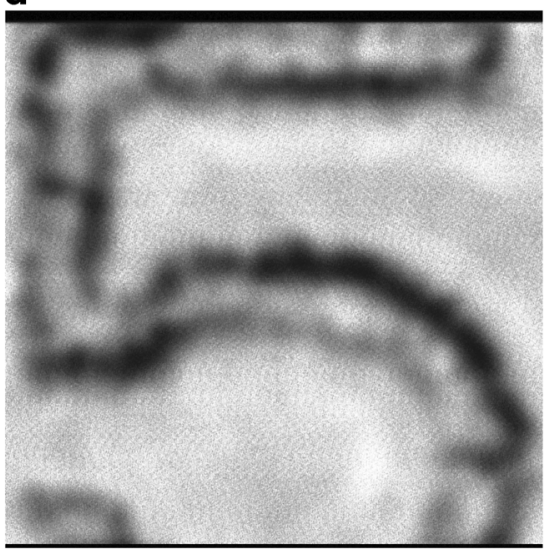




\subsection{Digital electronic image generation time}

Digital electronic image generation utilised a piece of $\mathrm{C} / \mathrm{C}++$ software receiving data from the data logger, i.e. Arduino DUE microcontroller break-out board (depicted in Fig. 1d), and a piece of Python software to conduct image pixel intensity allocation. Figure 6 is a screenshot from the computer running the software and displays a typical operational environment during electronic imaging generation. Table 6 summarises a typical set of image generation times. It shows that the typical total image generation time is less than $2 \mathrm{~s}$ with the settings described in Table 3.

\subsection{Digital image processing}

Raw digital images were generated from the electronic imaging experiments. Image noise was removed by applying a median filter, and image contrast was enhanced by carrying out histogram equalisation. Equation 1 [38] and Eq. 2 [38] define the median filter and histogram equalisation functions used. The median filter applied had a user-defined neighbourhood area of a circle with radius of 2 pixels. The histogram equalisation was carried out with a user-defined saturated pixel value of $0.3 \%$, allowing $0.3 \%$ of the total pixels to become saturated.

$\hat{f}(x, y)=\underset{(s, t) \in s_{x y}}{\text { median }}\{g(s, t)\}$

where

$\hat{f} \quad$ is the pixel value of the filtered image at $(\mathrm{x}, \mathrm{y})$

$(\mathrm{x}, \mathrm{y})$

$g(\mathrm{~s}, \mathrm{t}) \quad$ is the pixel value of the raw image at $(\mathrm{s}, \mathrm{t})$

$\mathrm{S}_{\mathrm{xy}} \quad$ represents the set of coordinates within a user-defined area of an image

$$
y_{\mathrm{k}} \triangleq\left\lfloor\left[(L-1) \sum_{i=0}^{k} h(i)\right]+0.5\right\rfloor \quad k=0,1,2, \ldots . ., L-1
$$

where

L is the bit depth in an image

$k \quad$ is the pixel value within the bit depth, $L$

$h(i) \quad$ is the normalised histogram which gives the

$k \quad$ probability of occurrence of pixel value, $i$
Fig. 8 a-d 1800 pixel $\times$ 1800 pixel electronic digital images (processed) of the four different $60 \mathrm{~mm} \times 60 \mathrm{~mm}$ imaging locations across the standard electronic imaging test build a

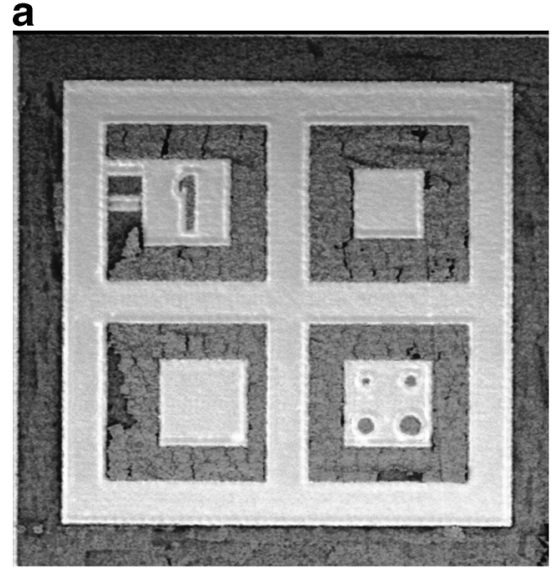

C

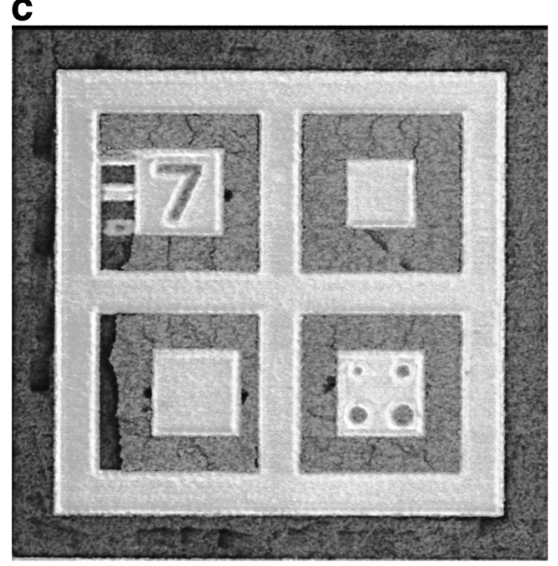

b

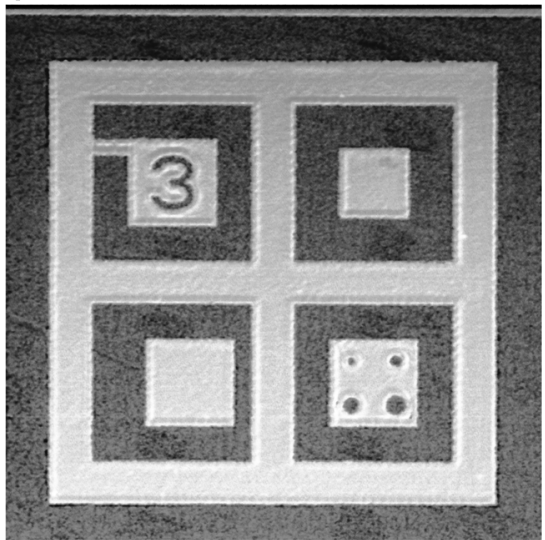

d

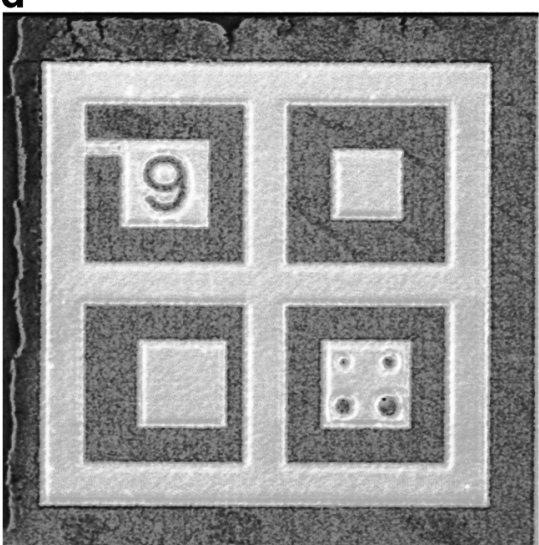


is the cumulative probability distribution of the normalised histogram

$y_{\mathrm{k}} \quad$ is an integer, the equalised number of pixel with a pixel value of $k$

\subsection{Image magnification}

A range of image magnifications was achieved in the first set of experiments. Table 7 gives details on the achievement and Fig. 7a-d shows a typical set of processed images.

\subsection{Image FOV}

The prototype also generated images from different FOVs in the second set of experiment. Nine images were generated from the nine imaging locations of the standard electronic imaging test build. Figure $8 \mathrm{a}-\mathrm{d}$ presents a selected set of processed images.

\subsection{Macroscopic process quality analysis software verification}

Figure 9a-c shows the "reference-virtual-workpiece" image set and resultant image from the verification. The virtual workpiece image, Fig. 9b, shows the artificial removal of the $8 \mathrm{~mm} \times 8 \mathrm{~mm}$ square on the top right-hand corner from

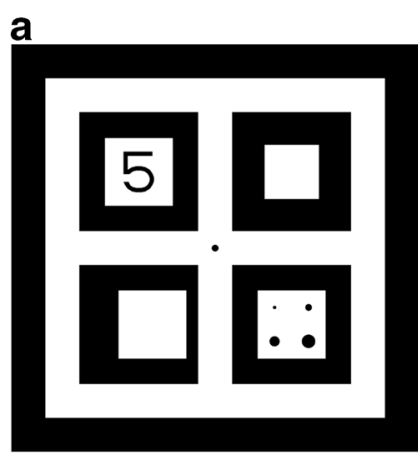

b

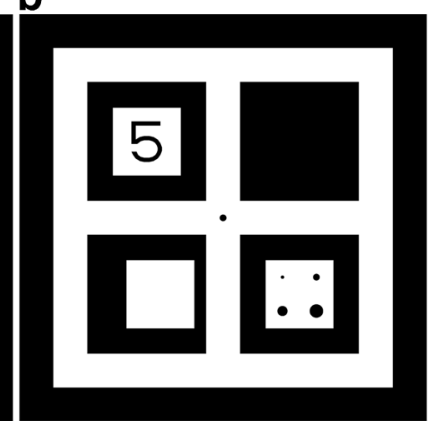

C

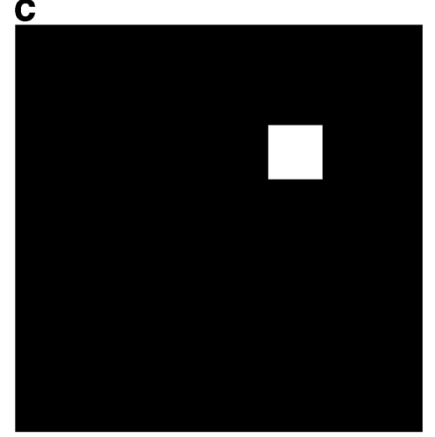

Fig. 9 Verification of the macroscopic process quality analysis software. a Reference image. b Virtual workpiece image. c Analysis resultant image with white pixels revealing the deviations between the virtual workpiece and reference image the reference image, Fig. 9a, as depicted in Fig. 5. Table 8 gives a quantitative summary of the macroscopic process quality analysis. The image size of the resultant image, Fig. $9 \mathrm{c}$, is 1800 pixel $\times 1800$ pixel, representing an image ROI of $60 \mathrm{~mm} \times 60 \mathrm{~mm}$ whilst the difference in the number of white pixels found is 57,600 . This difference in white pixel corresponds to an image area of $64 \mathrm{~mm}^{2}$ across the image ROI.

\subsection{Macroscopic process quality analysis demonstration}

Figure 10a shows a raw electronic workpiece image of the top surface of the build at location 5. This raw image has gone through noise removal and histogram equalisation by the application of Eqs. 1 and 2. After thresholding the processed image, the binary workpiece image, shown in Fig. 10b, has been generated. Figure 10c shows a binary reference image of the top layer of imaging location 5, covering the same image ROI as that of the workpiece image. It is extracted from the test build STL design. Macroscopic process quality analysis was carried out with the software and Fig. 10d is the resultant image. The white pixels in this bitmap reflect the deviations between the binary workpiece image and the binary reference image. Table 9 gives a quantitative summary of the macroscopic process quality analysis. The size of the resultant image, Fig. 10d, is 1800 pixel $\times 1800$ pixel, representing an image ROI of $60 \mathrm{~mm} \times 60 \mathrm{~mm}$ whilst the difference in the number of white pixels found is 31,891 . This difference in white pixel corresponds to an image area of $35.4 \mathrm{~mm}^{2}$ across the image ROI.

\section{Discussion}

In this section, discussions on image generation time, FOV, magnification, and the macroscopic process quality analysis are presented.

\subsection{Influence of image generation on EBAM process layer time}

Table 6 shows that the typical image generation time is $1.37 \mathrm{~s}$, and is additional to the EBAM process layer time. As EBAM build design varies, no single EBAM layer time could be used as a reference. In order to estimate the effect of the addition time attributing to image generation in a real-life situation, two different EBAM designs were brought in as a demonstrator design to carry out two sets of estimation. Table 10 summarises the EBAM build report obtained from the Arcam A1 EBAM machine and additional layer time. Figure 11a, b depicts the two designs. Results show that the additional time is 2.67 and $1.36 \%$ of the two selected demonstrator designs. Clearly, the additional time incurred by the use of electron imaging is a burden to the overall build time of the system; 
Table 8 Macroscopic process quality analysis verification results

\begin{tabular}{llllll}
\hline Image $\left(\right.$ size $\left./ \mathrm{mm}^{2}\right)$ & $\begin{array}{l}\text { Image shape } \\
\left(\text { pixel }^{2}\right)\end{array}$ & $\begin{array}{l}\text { Number of white } \\
\text { pixel }\end{array}$ & $\begin{array}{l}\text { Difference } \\
(\text { pixel })\end{array}$ & $\begin{array}{l}\text { Difference } \\
\left(\mathrm{mm}^{2}\right)\end{array}$ & $\begin{array}{l}\text { Process time } \\
(\mathrm{ms})\end{array}$ \\
\hline $\begin{array}{l}\text { Reference }(60 \times 60) \\
\begin{array}{l}\text { Virtual workpiece } \\
(60 \times 60)\end{array}\end{array}$ & $\begin{array}{l}1800 \times 1800 \\
1800 \times 1800\end{array}$ & $\begin{array}{l}1,459,609 \\
1,402,009\end{array}$ & 57,600 & 64 & 83 \\
\hline
\end{tabular}

however, the benefit of image generation outweighs such additional process layer time particularly when one considers the possibility of in-process build correction or process control.

\subsection{Image magnification}

The prototype was capable of generating images (Fig. 7a-d) of the test build with different user-defined magnifications. As Table 7 suggests, the image magnification is 2.6 when a $180 \mathrm{~mm} \times 180 \mathrm{~mm}$ area across the whole test build is imaged and the maximum magnification achieved is 95 when a $5 \mathrm{~mm} \times 5 \mathrm{~mm}$ area is imaged. The ability to achieve userdefined image magnification indicates that the prototype has the potential to zoom-in to specific ROIs, revealing local component features or defects when fully integrated with an EBAM machine.
Despite the achievement in image magnification, two issues can be observed in the images. Firstly, horizontal black stripes are detected at the top or at both the top and bottom in each of the resultant images (Fig. 7a-d). It is suspected that the stripes are caused by a delay in the EBAM machine electron gun to deliver the required beam current during experiment. With no interactions between the machine electron beam and the test build, no feedback electrons were generated and thus the prototype data logger registered no responses resulting in black stripes in the images. Apart from the delay, the second issue observed is the limitation in spatial resolution. In theory, if the machine electron beam is infinitely small, the image spatial resolution would increase with higher image magnification. In reality, the image spatial resolution cannot keep up with the increase in magnification, and thus results in visually blurry images (Fig. 7c, d). The blurriness implies that the electron beam diameter is larger than the pixel size in Fig.
Fig. 10 Demonstration of the macroscopic process quality analysis. a Electronic workpiece image (raw) of imaging location 5. b Processed and binarised workpiece image of location 5. c Binary electronic reference image of imaging location 5 , extracted from the STL design. d Analysis resultant image with white pixels revealing the deviations between the workpiece and the reference image a

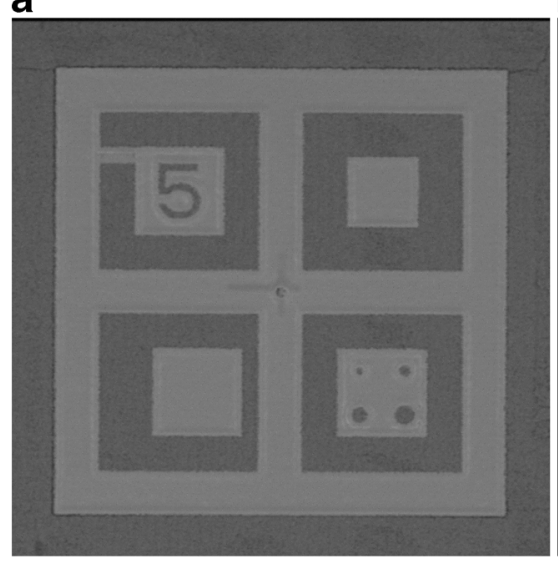

C

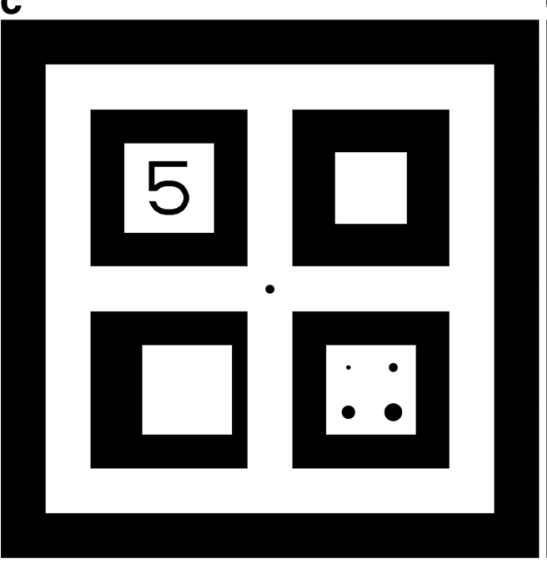

b

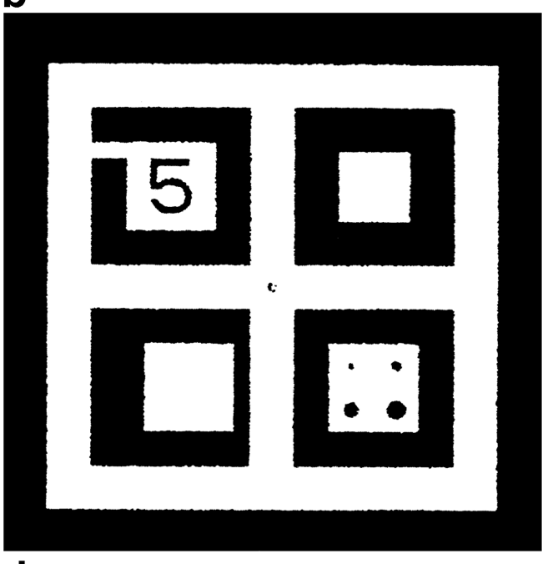

d

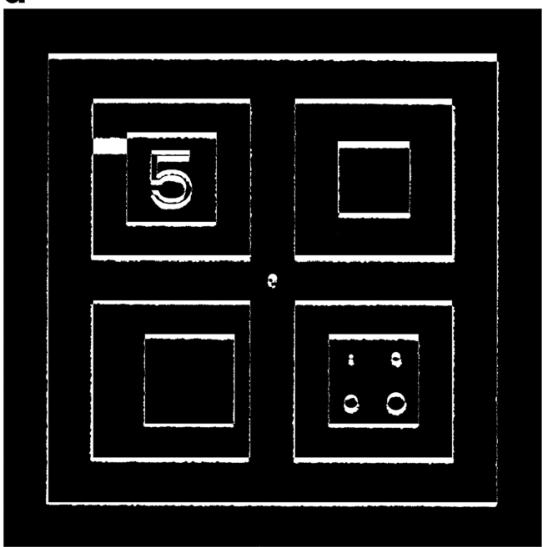


Table 9 Macroscopic process quality analysis results

\begin{tabular}{llllll}
\hline Image $\left(\right.$ size $\left./ \mathrm{mm}^{2}\right)$ & $\begin{array}{l}\text { Image } \\
\text { shape }\left(\text { pixel }^{2}\right)\end{array}$ & $\begin{array}{l}\text { Number of white } \\
\text { pixel }\end{array}$ & $\begin{array}{l}\text { Difference } \\
(\text { pixel })\end{array}$ & Difference $\left(\mathrm{mm}^{2}\right)$ & $\begin{array}{l}\text { Process } \\
\text { time }(\mathrm{ms})\end{array}$ \\
\hline $\begin{array}{c}\text { Reference } \\
(60 \times 60)\end{array}$ & $1800 \times 1800$ & $1,459,609$ & 31,891 & 35.4 & 123 \\
$\begin{array}{c}\text { Workpiece } \\
(60 \times 60)\end{array}$ & $1800 \times 1800$ & $1,427,718$ & & \\
\hline
\end{tabular}

7c, d, $5.56 \mu \mathrm{m} /$ pixel (3. s. f.) and $2.78 \mu \mathrm{m} /$ pixel (3 s. f.) respectively. The Arcam A1 EBAM specification [39] supports this, stating that the minimum achievable beam spot size (full-width-half-maximum) is $200 \mu \mathrm{m}$.

\subsection{Image FOV}

The prototype was capable of generating images (Fig. 8a-d) from the test build with different user-defined FOVs. The ability to achieve user-defined image FOV indicates that the prototype can allow specific ROI on the processing area to be imaged during future in-process EBAM monitoring. This ability is complementary to the imaging of the whole processing area. For the effective use of the machine processing area, multiple components of the same or different designs are always packed into the same EBAM build. Whilst an image of the whole processing area can provide information of the overall quality of an additive layer, an image of a specific ROI opens up opportunities to enable the effective analysis of individual components.

\subsection{Macroscopic process quality analysis software verification}

Verification was carried out on the macroscopic process quality analysis software. The virtual workpiece image (Fig. 9b) was overlapped on top of the reference image (Fig. 9a) and a resultant image (Fig. 9c) was produced. Table 8 shows that the difference between the reference and virtual workpiece image is 57,600 pixels, which corresponds to $64 \mathrm{~mm}^{2}$ (as $60 \mathrm{~mm}$ corresponds to 1800 pixel). This area is exactly the same as that covered by the artificially removed $8 \mathrm{~mm} \times 8 \mathrm{~mm}$ square on the top right-hand corner of the design depicted in Fig. 5. The result verifies the capability and correct operation of the software.

\subsection{Macroscopic process quality analysis demonstration}

Macroscopic process quality analysis demonstration was carried out on electronic images. The binary workpiece image (Fig. 10b) was overlapped on top of the binary reference image (Fig. 10c) and a resultant image (Fig. 10d) was produced. Table 9 shows that the difference between the reference and workpiece image is 31,891 pixels, which corresponds to $35.4 \mathrm{~mm}^{2}$ (as $60 \mathrm{~mm}$ corresponds to 1800 pixels). This demonstration gives a quantitative measure, serving as an indicator of the macroscopic process quality. Due to the wide range of variation on design and tolerance of different EBAM components, it is thought that no universal benchmark can be set using this macroscopic indicator, for the definition of good process quality.

There are two main root causes for the difference between the workpiece (Fig. 10b) and reference image (Fig. 10c). The first root cause is thought to be the misalignment between the test build location and the movements of the machine electron beam. In the demonstration, the test build was taken out of the chamber and put back manually for the removal of excess sintered powder. Due to the lack of fixation points on the EBAM machine processing area and the inaccuracy involved in manual handling, the location of the test build after powder removal was different from that when it was built, leading to a misalignment in the build location when electronic imaging experiments were carried out with the machine electron beam. The second root cause is thought to come from human error as well. Figure 10d shows that the left-hand side of the inner square containing the figure number 5 has the largest deviation. Referring to the binary workpiece image Fig. 10b, there is a horizontal bridge connecting the outer frame and the inner square. According to the binary reference image Fig. 10c, generated from the same ROI and layer height of the STL design as the workpiece image, the bridge should not be visible. The explanation is thought to be that the
Table 10 EBAM layer time and image generation time, data rounded to 3.s.f

\begin{tabular}{|c|c|c|c|c|c|}
\hline $\begin{array}{l}\text { EBAM } \\
\text { design }\end{array}$ & $\begin{array}{l}\text { Build time } \\
\text { (min) }\end{array}$ & $\begin{array}{l}\text { Number of } 50-\mu \mathrm{m} \text { layer } \\
\text { (build height } / \mathrm{mm} \text { ) }\end{array}$ & $\begin{array}{l}\text { Layer } \\
\text { time }(\mathrm{s})\end{array}$ & $\begin{array}{l}\text { Image } \\
\text { time (s) }\end{array}$ & $\begin{array}{l}\text { Ratio of image } \\
\text { time to layer } \\
\text { time }(\%)\end{array}$ \\
\hline 25 coupons & 605 & $706(35.3)$ & 51.4 & 1.37 & 2.67 \\
\hline Test build & 508 & $300(15)$ & 101 & 1.37 & 1.36 \\
\hline
\end{tabular}


Fig. 11 Top view of EBAM build designs. a 25 square coupons. b Ti-6Al-4V test build a Design at build height: $35 \mathrm{~mm}$

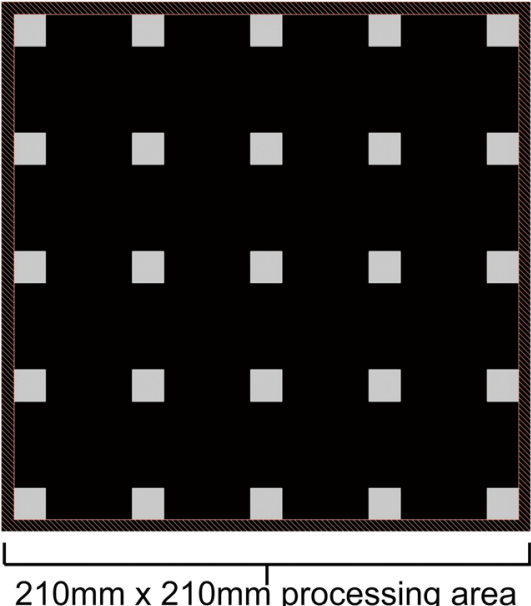

b Design at build height: $15 \mathrm{~mm}$

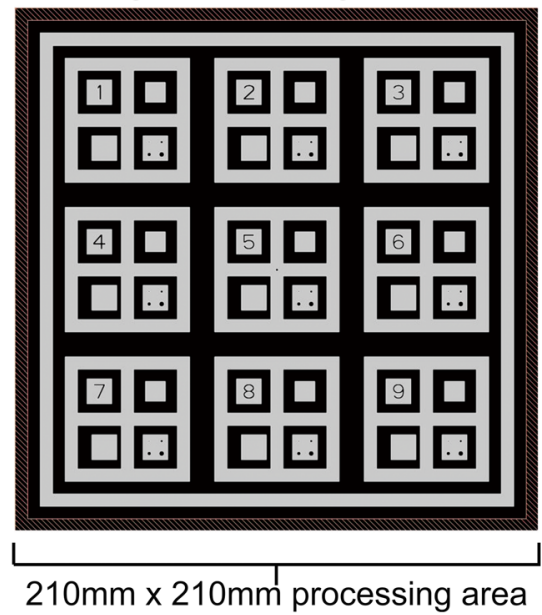

test build underwent excess sintered powder removal. Some of the sintered powder covering the top surface of imaging location 5 was unintentionally removed, thus revealing the subsurface horizontal melted bridge structure.

Unlike the demonstration, when the software is operating in a real, in-process EBAM monitoring condition, the workpiece image will be generated once the processing of a layer is complete. As a result, without any manual disturbances to the build location or the top surface of the processed layer, human errors will not contribute to the deviations observed. Any deviations measured in the macroscopic process quality analysis in a real setting will be due to errors and imperfections of the EBAM process. It is thought that the software could be used as an "imaging go/no-go gauge". It has the potential to detect process errors including over-melting, peeled-off metallisation [37], the lack of powder deposition in local processing area and undesired electron beam movement due to stray magnetic field. The described errors are expected to lead to a detectable difference in the macroscopic process quality analysis output. This software is thought to have the potential to contribute to the decision making of the acceptance or rejection of a processed layer by the EBAM machine.

\section{Conclusions}

Applications of an electronic imaging system prototype have been presented in this paper. The prototype was designed to interface with a commercial EBAM machine. With the use of the prototype, single-layer electronic imaging experiments were carried out with the EBAM machine at room temperature. Digital electronic images were generated. Four different magnifications were achieved with the maximum value being 95 . In addition, nine $60 \mathrm{~mm} \times 60 \mathrm{~mm}$ images with different FOVs were generated across the machine processing area. Moreover, macroscopic process quality analysis software was developed, verified and demonstrated. The demonstration showcased the capability of the software. It is thought that the software has the potential to be used for in-process EBAM macroscopic process quality analysis for individual components. With regard to in-process EBAM monitoring, it is thought that the electronic imaging system prototype has the potential to serve as an alternative to systems which employ thermal or optical imaging. There will be challenges moving to in-process monitoring in order to realise the systems potential. They are thought to include carrying out imaging on multi-layer for the whole additive manufacturing process, working at elevated temperature as the EBAM cycle includes preheating of the processing area and dealing with metallization generated from vaporisation of metal powder during the EBAM process.

Acknowledgements The EBAM machine was purchased, in part from a grant received for the EPSRC Centre for Innovative Manufacturing in Additive Manufacturing.

\section{Compliance with ethical standards}

Conflict of interest The authors declare that they have no conflict of interest.

Open Access This article is distributed under the terms of the Creative Commons Attribution 4.0 International License (http:// creativecommons.org/licenses/by/4.0/), which permits unrestricted use, distribution, and reproduction in any medium, provided you give appropriate credit to the original author(s) and the source, provide a link to the Creative Commons license, and indicate if changes were made.

Publisher's Note Springer Nature remains neutral with regard to jurisdictional claims in published maps and institutional affiliations.

\section{References}

1. Gibson I, Rosen DW, Stucker B (2010) Additive manufacturing technologies. Springer, New York, pp 126-130 
2. Gong X, Anderson T, Chou K (2014) Review on powder-based electron beam additive manufacturing technology. Manufacturing Review 1:2. https://doi.org/10.1051/mfreview/2014001

3. Harrysson OLA, Cansizoglu O, Marcellin-Little DJ, Cormier DR, West HA (2008) Direct metal fabrication of titanium implants with tailored materials and mechanical properties using electron beam melting technology. Mater Sci Eng C 28(3):366-373, ISSN 09284931. https://doi.org/10.1016/j.msec.2007.04.022

4. Baudana G, Biamino S, Ugues D, Lombardi M, Fino P, Pavese M, Badini C (2016) Titanium aluminides for aerospace and automotive applications processed by electron beam melting: contribution of Politecnico di Torino. Metal Powder Report 71(3):193-199, ISSN 0026-0657. https://doi.org/10.1016/j.mprp.2016.02.058

5. Jarow JP, Baxley JH (2015) Medical devices: US medical device regulation. Urologic Oncology: Seminars and Original Investigations 33(3):128-132, ISSN 1078-1439. https://doi.org/ 10.1016/j.urolonc.2014.10.004

6. Portolés L, Jordá O, Jordá L, Uriondo A, Esperon-Miguez M, Perinpanayagam S (2016) A qualification procedure to manufacture and repair aerospace parts with electron beam melting. J Manuf Syst 41:65-75, ISSN 0278-6125. https://doi.org/10.1016/j.jmsy. 2016.07.002

7. Mani M, Lane B, Donmez A, Feng S, Moylan S, Fesperman R (2015) NISTIR 8036. Measurement science needs for real-time control of additive manufacturing powder bed fusion processes. https://doi.org/10.6028/NIST.IR.8036

8. Raplee J, Plotkowski A, Kirka MM, Dinwiddie R, Okello A, Dehoff RR, Babu SS (2017) Thermographic microstructure monitoring in electron beam additive manufacturing. Sci Rep 7:43554. https://doi.org/10.1038/srep43554

9. Cordero PM, Mireles J, Ridwan S, Wicker RB (2017) Evaluation of monitoring methods for electron beam melting powder bed fusion additive manufacturing technology. Progress in Additive Manufacturing 2(1-2):1-10. https://doi.org/10.1007/s40964-0160015-6

10. Rodriguez E, Medina F, Espalin D, Terrazas C, Muse D, Henry C, MacDonald E, Wicker R (2012) Integration of a thermal imaging feedback control system in electron beam melting. Proceedings from the Solid Freeform Fabrication Symposium, pp 945-961

11. Price S,Cooper K, Chou K (2012) Evaluations of temperature measurmnets by near-infrared thermography in powder-based electron-beam additive manufacturing. Proceedings from the Solid Freeform Fabrication Symposium, pp 761-773

12. Price S, Lydon J, Cooper K, Chao K (2013) Experimental temperature analysis of powder-based electron beam additive manufacturing. Proceedings from the Solid Freeform Fabrication Symposium, pp $162-173$

13. Zalameda JN, Burke ER, Hafley RA, Taminger KMB, Domack CS, Brewer A, Martin RE (2013) Thermal imaging for assessment of electron-beam freeform fabrication additive manufacturing deposits. Proceeding of SPIE, Volume 8705, Thermosense: Thermal Infrared Applications XXXV, 87050M. https://doi.org/10.1117/12. 2018233

14. Scharowsky T, Bauereiß A, Singer RF, Körner C (2012) Observation and numerical simulation of melt pool dynamic and beam powder interaction during selective electron beam melting. Proceedings from the Solid Freeform Fabrication Symposium, Austin, pp 815-820

15. Ridwan S, Mireles J, Gayton SM, Espalin D, Wicker RB (2014) Automatic layerwise acquisition of thermal and geometric data of the electron beam melting process using infrared thermography. Proceedings from the Solid Freeform Fabrication Symposium, pp 343-352

16. Mireles J, Ridwan S, Motron PA, Hinojos A, Wicker RB (2015) Analysis and correction of defects within parts fabricated using powder bed fusion technology. Surface Topography: Metrology and Properties 3(3). https://doi.org/10.1088/2051-672X/3/3/ 034002

17. Dinwiddie RB, Dehoff RR, Lloyd PD, Lowe LE, Ulrich JUB (2013) Thermographic in-situ process monitoring of the electronbeam melting technology used in additive manufacturing. Proceeding of SPIE, Thermosense: Thermal Infrared Applications XXXV, 87050K. https://doi.org/10.1117/12.2018412

18. Schwerdtfeger J, Singer RF, Körner C (2012) In situ flaw detection by IR-imaging during electron beam melting. Rapid Prototyp J 18(4):259-263. https://doi.org/10.1108/13552541211231572

19. Mireles J, Terrazas C, Gaytan SM, Roberson DA, Wicker RB (2015) Closed-loop automatic feedback control in electron beam melting. Int J Adv Manuf Technol 78:1193-1199. https://doi.org/ 10.1007/s00170-014-6708-4

20. Mireles J, Terrazas C, Medina F, Wicker R (2013) Automatic feedback control in electron beam melting using infrared thermography. Proceedings from the Solid Freeform Fabrication Symposium, Austin, pp 708-717

21. Hobbs N (2017) Arcam EBAM, SE region aerospace supplier and advanced manufacturing summit presentation

22. National Institute of Standards and Technology U.S. Depeartment of Commerce (2013) Measurement science roadmap for metalbased additive manufacturing. Workshop Summary Report, pp 71

23. Tapia G, Elwany A (2014) A review on process monitoring and control in metal-based additive manufacturing. ASME Journal of Manufacturing Science and Engineering 136(6):060801-06080110. https://doi.org/10.1115/1.4028540

24. Everton SK, Hirsch M, Stravroulakis P, Leach RK, Clare AT (2016) Review of in-situ process monitoring and in-situ metrology for metal additive manufacturing. Mater Des 95:431-445, ISSN 0264-1275. https://doi.org/10.1016/j.matdes.2016.01.099

25. Grasso M, Colosimo BM (2017) Process defects and in situ monitoring methods in metal powder bed fusion: a review. Meas Sci Technol 28(4):044005. https://doi.org/10.1088/13616501/aa5c4f

26. Everton S (2015) In-process metrology for AM, Presentation

27. Oltean SE (2018) Strategies for monitoring and control with seam tracking in electron beam welding, Proceeding from $11^{\text {th }}$ International Conference Interdisciplinary in Engineering, INTER-ENG 2017, Procedia Manufacturing 22(2018):605-612

28. Trushnikov D, Krotova E, Koleva E (2016) Use of a secondary current sensor in plasma during electron-beam welding with Forus scanning for process control. Journal of Sensors 2016: 5302681. https://doi.org/10.1155/2016/5302681

29. Oltean SE, Abrudean M (2008) Advanced control of the electron beam welding. Journal of Control Engineering and Applied Informatics 10(1):40-48

30. Trushnikov D, Belenkiy V, Shchavlev V, Piskunov A, Abdullin A, Mladenov G (2012) Plasma charge current for controlling and monitoring electron beam welding with beam oscillation. Sensors 12(12):17433-17445. https://doi.org/10.3390/s121217433

31. Koleva EG, Mladenov GM, Trushnikov D, Belenkiy V (2014) Signal emitted from plasma during electron-beam welding with deflection oscillations of the beam. J Mater Process Technol 214(9):1812-1819. https://doi.org/10.1016/j.jmatprotec.2014.03. 031

32. Trushnikov D, Belenkiy V, Mladenov G, Portnov N (2012) Secondary-emission signal for weld formation monitoring and control in electron beam welding (EBW). Materialwissenschaft und Werkstofftechnik (Materials Science and Engineering Technology) 43(10):892-897. https://doi.org/10.1002/mawe. 201200933

33. Watt IM (1997) The principles and practice of electron microscopy. Cambridge University Press, Cambridge, pp 89-90. https://doi.org/ 10.1017/CBO9781139170529 
34. Oatley CW (1972) The scanning electron microscope. Cambridge University Press, Cambridge, pp 1-2

35. Wong H (2017) Pilot investigation of feedback electronic image generation in electron beam melting and its potential for inprocess monitoring. Elsevier Journal of Materials Processing Technology, in-press

36. Petrovic V, Haro JV, Blasco JR, Portolés L (2012) Additive manufacturing solutions for improved medical implants, Biomedicine, Dr. Chao Lin (Ed.), ISBN: 978-953-51-0352-3, InTech, pp 173
37. Tan X, Kok Y, Tor SB, Chua CK (2014) Application of electron beam melting (ebam) in additive manufacturing of an impeller, Proceeding of the $1^{\text {st }}$ International Conference on Progress in Additive Manufacturing. https://doi.org/10.3850/978-981-090446-3 076

38. Gonzalez RC, Woods RE (2008) Digital image processing, Pearson Education, Inc, pp 122-127, 322-327

39. Arcam A1 EBAM machine specification: http://www.arcam.com/ wp-content/uploads/Arcam-A1.pdf 